\title{
First validation of the PASSPORT training environment for arthroscopic skills
}

\author{
Gabriëlle J. M. Tuijthof · Maayke N. van Sterkenburg • Inger N. Sierevelt • \\ Jakob van Oldenrijk · C. Niek Van Dijk · Gino M. M. J. Kerkhoffs
}

Received: 10 April 2009/ Accepted: 29 June 2009/Published online: 24 July 2009

(C) Springer-Verlag 2009

\begin{abstract}
The demand for high quality care is in contrast to reduced training time for residents to develop arthroscopic skills. Thereto, simulators are introduced to train skills away from the operating room. In our clinic, a physical simulation environment to Practice Arthroscopic Surgical Skills for Perfect Operative Real-life Treatment (PASSPORT) is being developed. The PASSPORT concept consists of maintaining the normal arthroscopic equipment, replacing the human knee joint by a phantom, and integrating registration devices to provide performance feedback. The first prototype of the knee phantom allows inspection, treatment of menisci, irrigation, and limb stressing. PASSPORT was evaluated for face and construct validity. Construct validity was assessed by measuring the performance of two groups with different levels of arthroscopic experience (20 surgeons and 8 residents). Participants performed a navigation task five times on PASSPORT. Task times were recorded. Face validity was assessed by completion of a short questionnaire on the participants' impressions and comments for improvements. Construct validity was demonstrated as the surgeons (median task time $19.7 \mathrm{~s}$ [8.0-37.6]) were more efficient than the residents (55.2 s [27.9-96.6]) in task completion for each repetition (Mann-Whitney $\mathrm{U}$ test, $P<0.05$ ). The
\end{abstract}

G. J. M. Tuijthof $(\varangle) \cdot$ M. N. van Sterkenburg

I. N. Sierevelt · J. van Oldenrijk · C. N. Van Dijk ·

G. M. M. J. Kerkhoffs

Orthopedic Research Center Amsterdam,

Department of Orthopedic Surgery, Academic Medical Centre,

Meibergdreef 9, 1105 AZ Amsterdam, The Netherlands

e-mail: g.j.tuijthof@amc.uva.nl; g.j.m.tuijthof@tudelft.nl

G. J. M. Tuijthof

Department of Biomechanical Engineering, Delft University of Technology, Mekelweg 2, 2628 CD Delft, The Netherlands prototype of the knee phantom sufficiently imitated limb outer appearance (79\%), portal resistance (82\%), and arthroscopic view (81\%). Improvements are required for the stressing device and the material of cruciate ligaments. Our physical simulation environment (PASSPORT) demonstrates its potential to evolve as a training modality. In future, automated performance feedback is aimed for.

Keywords Arthroscopy · Skills · Simulator · Training · Validity $\cdot$ Knee

\section{Introduction}

Arthroscopic surgery has become the leading operative therapy for a growing number of injuries. Learning arthroscopic skills presents several challenges because of reduced visibility, reduced degrees of freedom of instruments, different eye-hand coordination, loss of force feedback, and loss of tactile feedback [2, 7, 20, 24, 25]. Therefore, the majority of these techniques demand a significant amount of surgical skills [13, 15, 17]. This results in relatively long learning curves to acquire arthroscopic skills and many hours of training [6, 14, 18]. As the importance of training surgical skills is acknowledged, methods are introduced for training arthroscopic skills away from the operating room $[3,8,11]$. This is especially important, because in a considerable number of centers training time for residents is limited [4, 11]. By first training in a simulation environment until a certain level of skills is achieved, the risk of surgical errors is decreased when a resident is training in the real-life setting. This way patient safety will be secured to a larger extent.

Traditionally, cadaveric material is used as the best available model of human joints as it closely resembles 
in vivo tissue and replicates clinical variation. Its importance is illustrated by the many cadaveric hands-on workshops that are offered. On the other hand, cadaveric material has limited possibilities to provide feedback on performance and is not always available [9, 15]. More recently, several virtual reality simulators were introduced, inspired by the airline industry, which has embraced this technology for decades $[6,14,20,21]$. Virtual reality trainers have high educational potential due to their capability of providing visual feedback combined with direct feedback on performance after a training session [11]. However, the recreation of realistic tissue behavior as well as force and tactile feedback remains challenging in these trainers [28]. Especially, this force and tactile feedback is important in arthroscopic surgery. Lastly, various physical models of joints have become commercially available offering standardized anatomic features such as ruptured menisci for the knee joint [10, 19]. Drawbacks of these models are their lack of arthroscopic reality, which makes training insufficiently challenging, and lack of registration devices to offer feedback on performance [15]. A strong advantage of the physical models is the presence of inherently natural tactile feedback when manipulating the instruments in the limbs. The importance of these latter features in training of arthroscopic skills is obvious and previously demonstrated by Moody et al. [16]. At present, none of the simulators fulfils the three main criteria of providing sufficient clinical variation, natural sensory feedback (i.e., visual and force feedback), and direct feedback on performance into one system [12].

Our purpose was to combine the strong features of virtual reality systems and physical models into one design: a physical simulation environment to Practice Arthroscopic Surgical Skills for Perfect Operative Real-life Treatment (PASSPORT) [26], that offers all three requirements. The present study is to present our first prototype of PASSPORT among potential users. Secondly, the aim was to determine its face and construct validity for a navigation task as has been demonstrated for several virtual reality simulators $[6,14,20]$. This way it is potential to evolve as training modality will be assessed.

\section{Method and materials}

The concept of PASSPORT aims to imitate the surgical setting as realistically as possible. This has been achieved by using standard arthroscopic equipment with a $30^{\circ}$ $\varnothing 4 \mathrm{~mm}$ arthroscope; the human joint is replaced with a phantom version. Additionally, registration devices were integrated to provide feedback and registration of training sessions. An arthroscopic training routinely starts with surgery in the knee. The knee is the joint most frequently treated with arthroscopy. Therefore, the first focus was on the development of a knee phantom $[1,26]$. The challenge was to design the phantom realistically, i.e., its appearance had to be anatomically correct and it had to enable realistic surgical actions. The construction included basic anatomic structures that match human shape and geometry; the three most commonly used access portals (anteromedial, anterolateral, and superomedial portal) [5, 23]; allowed menisci to be punched; and simulated disturbances in the arthroscopic view (e.g., bleeding or air bubbles) (Fig. 1). The creamy colored silicone menisci could be replaced by new pairs to start a new training session. The cruciate ligaments were made of white colored woven rope and anatomically attached (Fig. 2). The patellofemoral structures were not included at this stage. The knee phantom's irrigation behavior was validated in a previous study [26]. Additionally, a device for stressing the joint was constructed with medial and lateral springs and a steel tube representing the collateral ligaments and the lower limb, respectively. The preload of the springs could be adjusted to decrease or increase the amount of joint laxity. In Fig. 1a, a participant performs a stressing action on PASSPORT with her right thigh. Finally, a foam cover was added over the complete construction to indicate the $90^{\circ}$ angular position of the lower leg related to the upper leg (Fig. 1). The registration devices (VISION DAQ v1.2, MTO, Amsterdam, the Netherlands) enabled synchronized acquisition of four data channels and two digital video streams: the view in the knee joint from underneath a transparent tibial surface, and the arthroscopic view [26].

During the international conference of the Nordic Orthopedic Federation in June 2008, participants were recruited to perform validity tests in a skills lab. Twentyeight participants completed the experiment. The group comprised of 20 surgeons and 8 residents. Fifteen surgeons had completed more than 50 arthroscopies annually in the past 5 years and five had completed between 20 and 50 arthroscopies annually in the past $2-5$ years. The residents had completed less than 20 arthroscopies annually in the past 2 years.

Construct validity of PASSPORT was assessed by measuring the performance of the two groups with different levels of arthroscopic experience (surgeons verus residents) $[14,27]$. We investigated whether PASSPORT could discriminate between the groups. Participants were asked to perform a navigation task in PASSPORT (Fig. 2) using normal arthroscopic equipment (Arthrex Nederland bv, Sint Anthonis, The Netherlands). The scope was placed in the anterolateral portal and the probe in the anteromedial portal. Nine landmarks had to be identified by probing (Fig. 2). The task time was defined as the time between the moment of first probing of the medial femoral condyle and the moment of first probing of the mid-section of lateral 

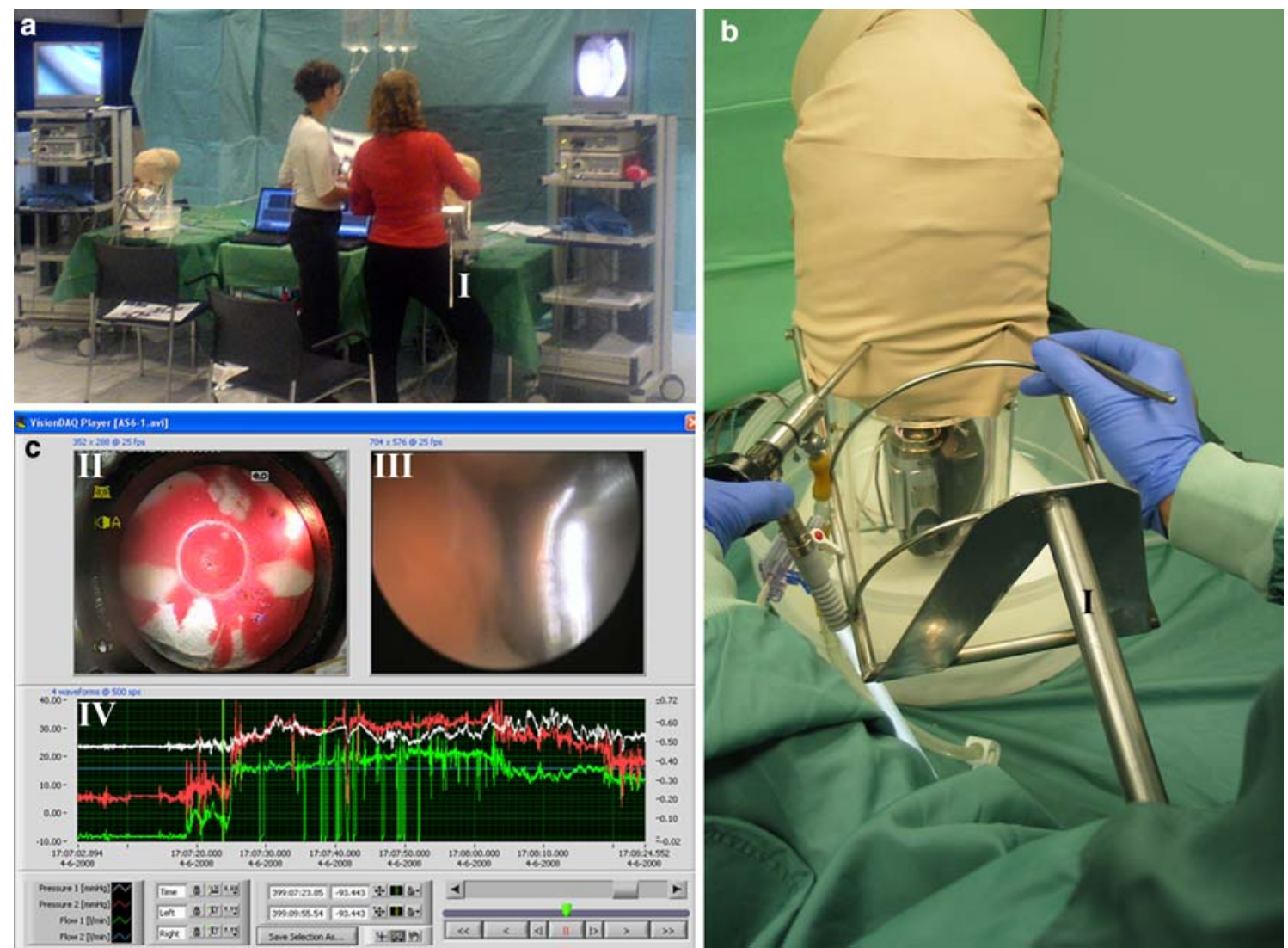

Fig. 1 a Setup of the skills lab during the experiment with two PASSPORTs. b Close up of the prototype of PASSPORT showing the imitation of the lower leg (I) and the foam cover. c Interface of the VisionDAQ software showing the view on the knee joint from underneath the tibial surface (II), the arthroscopic view with a metal

meniscus, where each of the other landmarks had to be probed in the described sequence (Fig. 2). Both the view from the arthroscope and from underneath the transparent tibial plateau were recorded. The task time was determined from the video recording of the arthroscope, assisted by the other view. Accurate determination of the task time was ensured, since the video recordings had a frame rate of 25 images per second and allowed replay frame by frame. This resulted in an accuracy of $0.04 \mathrm{~s}$. The purpose was to objectify the familiarization process to the PASSPORT system. Therefore, the surgeons were not allowed to familiarize with PASSPORT before the experiment, as this could introduce bias. Instead, they were asked to repeat the navigation task five times to assess a learning curve. This way the process of familiarization was highly conditioned.

This number of trials was determined by previous measurements using a virtual reality simulator which showed that surgeons needed approximately five attempts to reach a learning curve plateau [27]. During the navigation task, joint irrigation was possible with a gravity pump and suction (Fig. 1). To demonstrate an extra feature of probe (III), and two pressure (white and red) and one flow (green) signals (IV). In this case a bleeding has been injected. The surgeon controls the bleeding by intermittently opening and closing of the sheath as is demonstrated by the green flow signal (IV)

PASSPORT, participants were randomly assigned to experience a bleeding during their fifth repetition (Fig. 1c). Randomization was performed by a computer program Matlab, version 7.0.4.365 (R14) (The Mathworks, Natick, USA) that assigned randomly a 1 (no bleeding) or 2 (bleeding) to each participant.

To assess the face validity of PASSPORT, all 28 surgeons were asked to answer three questions before the experiment to give their opinion on the visual appearance of PASSPORT (Table 1). Afterwards, the participants were asked to complete another questionnaire to provide feedback on their impression and give free text suggestions for improvements (Table 2).

Data were processed with the special VISION DAQ Player and Microsoft Office Excel 2003 (Microsoft Corporation, Redmond, Washington, USA). One observer determined all navigation task times using the recorded arthroscopic view by consecutive determination of each identified landmark. This way, the proper sequence in the landmark identification protocol was guaranteed. One complete task time was calculated as the summation of the 
Fig. 2 Pictures of the intraarticular joint space of PASSPORT. The landmarks had to be probed in the following sequence for the navigation task. a medial femoral condyle, $\mathbf{b}$ medial tibial plateau, c posterior horn of medial meniscus, $\mathbf{d}$ mid-section of medial meniscus, $\mathbf{e}$ anterior cruciate ligament, $\mathbf{f}$ lateral femoral condyle, $\mathbf{g}$ lateral tibial plateau, $\mathbf{h}$ posterior horn of lateral meniscus, and $\mathbf{i}$ midsection of lateral meniscus

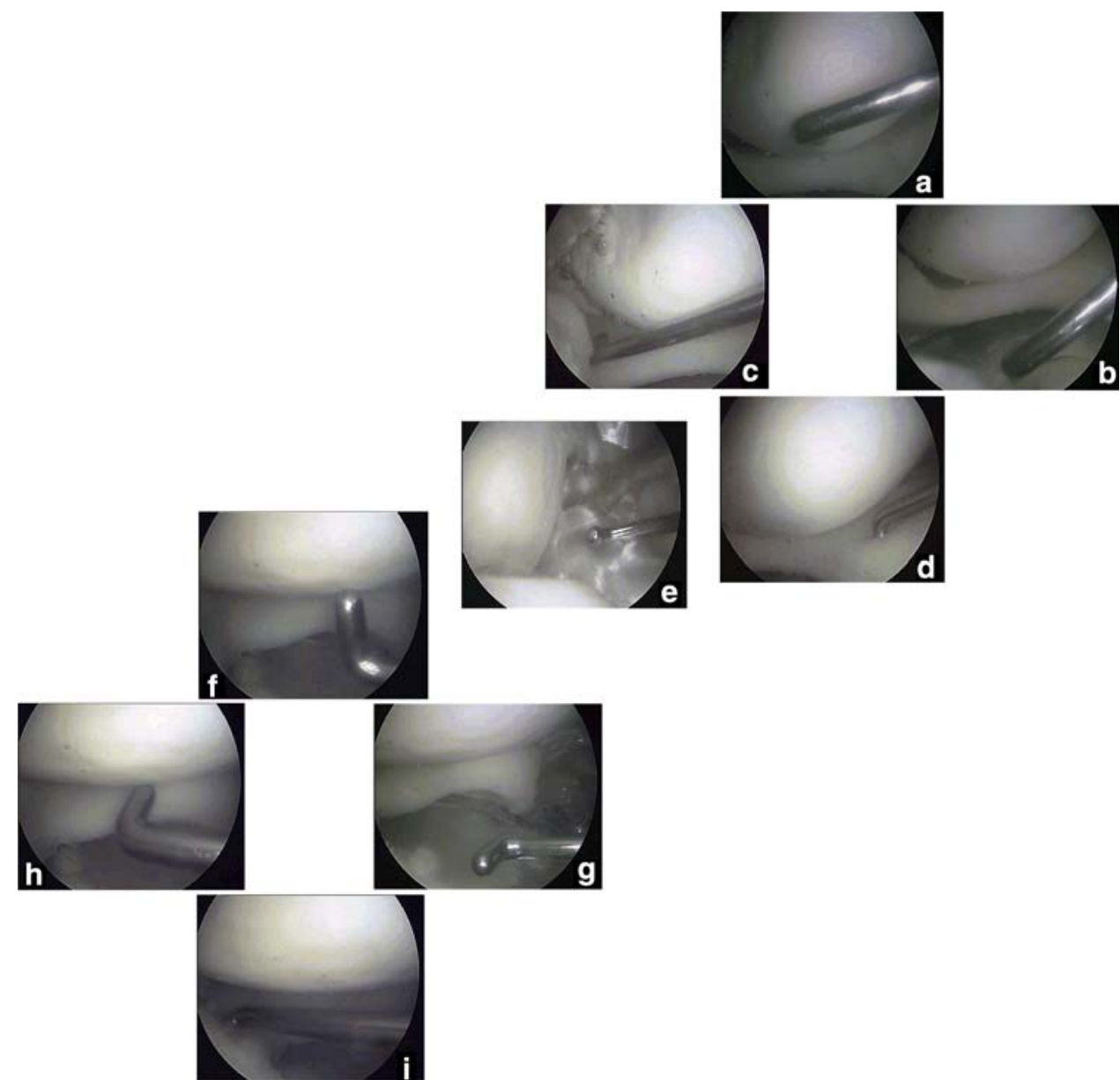

Table 1 Questions and results on the outer appearance of PASSPORT

1. What is your opinion on the outer appearance of PASSPORT? Not important, the intra-articular anatomy is what matters 9 The outer appearance is sufficient, because The outer appearance is insufficient, because

2. How do you recognize that PASSPORT is a physical knee phantom on the outside?

Portals

Color of PASSPORT

Shape of PASSPORT

Positioning of PASSPORT relative to the operational equipment 10

Equipment surrounding PASSPORT

$$
\text { Yes No }
$$

3. Is it clear how you can stress PASSPORT to increase the $23 \quad 5$ intra-articular joint space?

The numbers represent the participants. Multiple answers were allowed for question 2

duration between all landmarks of one trial. Statistical analysis was performed using SPSS 15 (SPSS Inc., Chicago, IL, USA). Due to a skewed distribution and a small sample size of the residents, nonparametric tests were
Table 2 Questionnaire and results concerning the experience with PASSPORT

\begin{tabular}{lrr}
\hline Question & Yes & No \\
\hline 1. Did the instrument movement in the portal (portal & 23 & 5 \\
resistance) imitate reality sufficiently? & 22 & 5 \\
2. Did the arthroscopic image itself imitate reality & & \\
sufficiently? & 17 & 10 \\
3. Was the intra-articular anatomy sufficiently imitated? & 25 & 3 \\
4. Was the knee joint space sufficiently imitated? & 15 & 12 \\
5. Was the device for stressing the knee sufficiently & & \\
imitated? & 26 & 2 \\
6. Does PASSPORT allow training of knee joint & & \\
inspection? & 15 & 10 \\
7. Does PASSPORT allow training of joint irrigation? & 25 & 0 \\
8. Does PASSPORT allow training of meniscectomy? & 27 & 0 \\
9. Do you think PASSPORT has potential to evolve as & 27 \\
training modality? & &
\end{tabular}

The numbers represent the participants. In some cases not all participants answered all questions

performed. Construct validity was assessed by calculation of significant statistical differences in task time between the two groups with the nonparametric Mann-Whitney U 


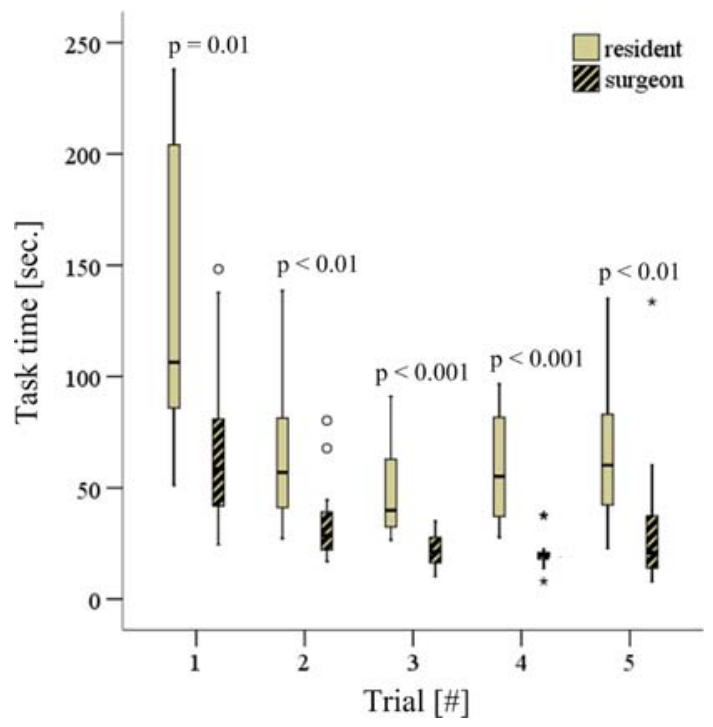

Fig. 3 Results of the task times of the navigation trials for each of the five repetitions. The task times are shown in the form of box-plots where the circles and asterisks indicate outliers, and the thick horizontal line in the boxes are the median task times. The box-plots indicate that a number of task times are not normally distributed

test. The alpha significance level was corrected for multiple testing and set at 0.01 (Bonferroni correction). Values were presented as median (minimum-maximum).

\section{Results}

The median task time of the first trial was $58.6 \mathrm{~s}$ (24.5$148.3 \mathrm{~s})$ for the surgeons and $106.4 \mathrm{~s}(51.2-406.0 \mathrm{~s})$ for the residents, respectively. The median task time of the fourth trial was $19.7 \mathrm{~s}(8.0-37.6 \mathrm{~s})$ for the surgeons and $55.2 \mathrm{~s}$ (27.9-96.6 s) for the residents, respectively. The median task time of the fifth trial was $20.9 \mathrm{~s}(8.0-133.5 \mathrm{~s})$ for the surgeons and $60.2 \mathrm{~s}(22.7-135.2 \mathrm{~s})$ for the residents, respectively. The median duration of the fifth trial was higher than that of the fourth, because a bleeding was randomly offered to half of the participants in the fifth trial time (Fig. 1c). The median time of all participants without bleeding was $19.8 \mathrm{~s}$ and with bleeding $27.8 \mathrm{~s}$. For the navigation task, the surgeons were faster in task completion than the residents. The difference in task time was significant for every trial $(P \leq 0.01)$ (Fig. 3).

Not all questions were answered by all participants (Tables 1,2). The combination of color, shape, portals, and positioning of PASSPORT relative to the equipment indicates the purpose of the training environment (Table 1). The outer appearance of PASSPORT is indicated as not important by $32 \%$ of the participants, and classified as sufficient by $79 \%$ of the participants who do think it is important (Table 1). The function of the stressing device was understood from inspection alone (Table $1 ; 82 \%)$. The participants generally agree portal resistance $(82 \%)$, the arthroscopic view (81\%), and the intra-articular joint space $(63 \%)$ are sufficiently imitated (Table 2). Suggestions are posed to choose other material for the cruciate ligaments as the woven rope tends to unwind (eight participants). Four participants are distracted by the reflection of the transparent tibial surface. The stressing device needs improvement according to 12 participants $(56 \%)$, as normally more force is used to stress the knee and the effect of stressing is more visible in reality. A majority agrees that PASSPORT can be used to train knee joint inspection and navigation (93\%), and to train meniscectomy (100\%), even though a meniscectomy was not performed during the experiment (Table 2). This implies that the participants could imagine using PASSPORT to train meniscectomy. The usefulness of PASSPORT for specific training on efficient irrigation to clear the view is less recognized (54\%). This is predominantly due to the suction device that did not provide sufficient vacuum pressure (four participants). All participants indicate that PASSPORT has potential to evolve as training modality and can be optimally applied in the first year of the residency curriculum prior to the first arthroscopy on a patient. Participants suggest continuing training with PASSPORT until approximately the first 20 arthroscopies are performed. A preference list for requested training possibilities includes: meniscectomy (13 participants), inspection, i.e., a-traumatic navigation, triangulation, and improving eye-hand coordination (12 participants), tunnel placement for anterior cruciate ligament reconstruction six participants), irrigation (two participants), removal of loose bodies (two participants), and portal placement (one participant).

\section{Discussion}

The most important finding of the present study was that, the PASSPORT prototype shows face and construct validity. The development of a physical simulation environment to train arthroscopic skills is clinically relevant as it offers the possibility to integrate three main requirements for an optimal simulator and could eventually replace part of the cadaveric material for training. This way, residents can be well prepared in a possibly shorter period before they are allowed to act in the operating room. This approach would enhance patient safety as well.

Although the PASSPORT knee phantom has a somewhat bulky appearance, a large majority of the surgeons who do care about the outer appearance indicate that it is sufficient, especially when placed in real-life operative surroundings (Table 1). The arthroscopic view inside the PASSPORT knee phantom is judged as sufficient by all 
participants. They indicate that the possibility of irrigation and generation of bleedings enlarges the sense of realism of PASSPORT. The transparency of the tibial surface causes reflections that can be confusing at times. For this study, the camera underneath the tibial surface was mainly used as an extra means to verify the proper execution of the navigation task, which might not outweigh the disturbance of the reflections. However, our future plans are to use the tibial surface video images to determine performance parameters and to assess its value for first time trainees in visual orientation in the knee phantom.

In addition to the visualization, the presence of tactile feedback in an arthroscopy simulator was considered essential to imitate clinical practice adequately [16, 29]. PASSPORT offers this type of feedback. Both the portal resistance and the probing of anatomic structures were sufficiently imitated, but there is need for improvement of the stressing device (Table 1).

Construct validity was clearly demonstrated by the significant difference in navigation task times between the surgeons and the residents. The experts show lower task times in every trial and a smaller variance after the initial trial.

A limitation of our study is that the task time was not automatically processed and the number of unallowed tissue contacts was not determined. Additionally, not all questionnaires were completed. However, the possible bias introduced by this was expected to be minimal. First, the questions were not systematically missed by the participants, but a lot of participants 'forgot' to fill out one question randomly. Second, only three participants at the most did not fill out the question (Table 2, Questions 7 and 8), which is a small number compared to the total group of 28. The study was performed during a conference, which could have introduced a selection bias, since only participants could have participated who had an intrinsic interest in training modalities. If this was the case, it is expected that they might have been more critical, but also be more motivated to execute the tasks at their best.

To the knowledge of the authors no comparable physical simulation environment exists. Thereto, comparison was performed with virtual reality arthroscopy simulators. They show similar high discriminative capability between groups of different levels of expertise [14, 20]. As the time was limited during the conference, we were not able to test a meniscectomy or removal of a loose body in PASSPORT. Replacement of a pair of menisci is, however, possible and is fairly quickly performed (less than $5 \mathrm{~min}$ ). In a future study, the inherent natural tactile feedback when performing these latter treatments can show the value of PASSPORT opposed to virtual reality simulators $[6,14$, $20,21]$.
All participants indicate that PASSPORT has the potential to evolve as a valuable training modality. The majority of requested training steps as indicated by the participants can be met with the current prototype. These training steps are in agreement with a previous study by Safir et al. [22] who found that identification of anatomic structures and navigation of the arthroscope are the most important skills to learn. More specifically, triangulating the tip of the probe with a $30^{\circ}$ scope, identification of lateral compartment, identification of medial compartment, and identification of intercondylar notch, including ACL and PCL are the four suggested arthroscopic skills to be trained before a resident starts operating on a patient [22]. Each of those skills can be trained in PASSPORT.

\section{Conclusion}

With these encouraging results, we conclude that PASSPORT can evolve to a modern physical training simulation environment able to improve residents' surgical skills prior to knee arthroscopy in patients. Future improvements will be automated performance assessment, improve the material of cruciate ligaments and stressing devices, and redesign the PASSPORT knee phantom to allow portal placement and tunnel placement for cruciate ligament reconstructions.

Acknowledgments This work was supported by the Technology Foundation STW, applied science division of NWO and the technology program of the Ministry of Economic Affairs, the Netherlands and by the Dutch Association of Arthroscopy (NVA). Arthrex Nederland bv (Sint Anthonis, the Netherlands) provided all arthroscopic equipment for the evaluation. The authors wish to thank D.T. Nguyen and all the medical students that assisted during the experiments as well as Marieke van Nieuwenhuizen for her help in the construction of PASSPORT. Finally, we want to give special thanks to all the orthopedic surgeons and residents that participated in the experiment during the Nordic Orthopedic Federation conference in June 2008.

\section{References}

1. Baker B, Lubowitz J. Meniscus injuries. eMedicine. Available at http://www.emedicine.com/sports/topic160.htm. Accessed 24 Aug 2007

2. Barrett DS, Green RG, Copeland SA (1991) Arthroscopic and endoscopic skills: a method of assessment. Ann R Coll Surg Engl 73:100-104

3. Bliss JP, Hanner-Bailey HS, Scerbo MW (2005) Determining the efficacy of an immersive trainer for arthroscopy skills. Stud Health Technol Inform 111:54-56

4. Bridges M, Diamond DL (1999) The financial impact of teaching surgical residents in the operating room. Am J Surg 177:28-32

5. Brophy R, Dunn W, Wickiewicz T (2004) Arthroscopic portal placement. Tech Knee Surg 3:2-7

6. Cannon WD, Eckhoff DG, Garrett WE Jr, Hunter RE, Sweeney HJ (2006) Report of a group developing a virtual reality simulator 
for arthroscopic surgery of the knee joint. Clin Orthop Relat Res 442:21-29

7. de Visser H, Heijnsdijk EA, Herder JL, Pistecky PV (2002) Forces and displacements in colon surgery. Surg Endosc 16:1426-1430

8. Gomoll AH, Pappas G, Forsythe B, Warner JJ (2008) Individual skill progression on a virtual reality simulator for shoulder arthroscopy: a 3-year follow-up study. Am J Sports Med 36:1139-1142

9. Grechenig W, Fellinger M, Fankhauser F, Weiglein AH (1999) The Graz learning and training model for arthroscopic surgery. Surg Radiol Anat 21:347-350

10. Hillway Surgical Ltd. Advanced models for simulated surgery. Chichester, UK. Available at http://www.surgimodels.com/ default.htm. Accessed 4 Jan 2008

11. Howells NR, Gill HS, Carr AJ, Price AJ, Rees JL (2008) Transferring simulated arthroscopic skills to the operating theatre: a randomised blinded study. J Bone Joint Surg Br 90:494499

12. Issenberg SB, McGaghie WC, Petrusa ER, Lee GD, Scalese RJ (2005) Features and uses of high-fidelity medical simulations that lead to effective learning: a BEME systematic review. Med Teach 27:10-28

13. Mabrey JD, Cannon WD, Gillogly SD, Kasser JR, Sweeney HJ, Zarins B, Mevis H, Garrett WE, Poss R (2000) Development of a virtual reality arthroscopic knee simulator. Stud Health Technol Inform 70:192-194

14. McCarthy AD, Moody L, Waterworth AR, Bickerstaff DR (2006) Passive haptics in a knee arthroscopy simulator: is it valid for core skills training? Clin Orthop Relat Res 442:13-20

15. Meyer RD, Tamarapalli JR, Lemons JE (1993) Arthroscopy training using a "black box" technique. Arthroscopy 9:338-340

16. Moody L, Waterworth A, McCarthy AD, Harley P, Smallwood R (2008) The feasibility of a mixed reality surgical training environment. Virtual Real 12:77-86

17. Morris AH, Jennings JE, Stone RG, Katz JA, Garroway RY, Hendler RC (1993) Guidelines for privileges in arthroscopic surgery. Arthroscopy 9:125-127
18. O'Neill PJ, Cosgarea AJ, Freedman JA, Queale WS, McFarland EG (2002) Arthroscopic proficiency: a survey of orthopaedic sports medicine fellowship directors and orthopaedic surgery department chairs. Arthroscopy 18:795-800

19. Pacific Research Laboratories. Sawbones. Vashon, WA, USA. Available at http://www.sawbones.com/. Accessed 3 Jan 2007

20. Pedowitz RA, Esch J, Snyder S (2002) Evaluation of a virtual reality simulator for arthroscopy skills development. Arthroscopy 18:E29

21. Riener R, Frey M, Proll T, Regenfelder F, Burgkart R (2004) Phantom-based multimodal interactions for medical education and training: the Munich Knee Joint Simulator. IEEE Trans Inf Technol Biomed 8:208-216

22. Safir O, Dubrowski A, Mirsky L, Lin C, Backstein D, Carnahan A (2008) What skills should simulation training in arthroscopy teach residents? Int J Comput Assist Radiol Surg 3(5):1-5

23. Sisk TD (1987) Arthroscopy of knee and ankle. In: Crenshaw AH (ed) Campbell's operative orthopedics. CV Mosby Company, St. Louis, pp 2547-2608

24. Sjoerdsma W (1998) Surgeons at work: time and actions analysis of the laparoscopic surgical process. Dissertation, Delft University of Technology, Delft, The Netherlands

25. Tuijthof GJM (2003) Technical improvement of arthroscopic techniques. Dissertation, Delft University of Technology, Delft, The Netherlands

26. Tuijthof GJM, Heeman P, Van Dijk CN, Blankevoort L (2009) Physical simulation environment for arthroscopic joint irrigation. J Med Devices 3:1-6

27. Verdaasdonk EG, Stassen LP, Schijven MP, Dankelman J (2007) Construct validity and assessment of the learning curve for the SIMENDO endoscopic simulator. Surg Endosc 21:1406-1412

28. Wentink M, Stassen LP, Alwayn I, Hosman RJ, Stassen HG (2003) Rasmussen's model of human behavior in laparoscopy training. Surg Endosc 17:1241-1246

29. Zivanovic A, Dibble E, Davies B, Moody L, Waterworth A (2003) Engineering requirements for a haptic simulator for knee arthroscopy training. Stud Health Technol Inform 94:413-418 